\title{
Patient with Syphilitic Thoracic and Abdominal Aortic Aneurysms
}

\author{
Kaspars Kisis*, Dainis Krievins*, Marcis Gedins*, Janis Savlovskis**, Natalija Ezite**, Patricija Ivanova *** \\ * Pauls Stradins Clinical University Hospital, Department of Vascular surgery, Latvia \\ ** Pauls Stradins Clinical University Hospital, Institute of Radiology, Latvia \\ *** University of Latvia
}

\section{SUMMARY}

We are presenting a rare case of patient with two syphilitic aneurysms localized in thoracic and abdominal aorta. Routine lung computer tomography (CT) for the patient with complains about irritating and unclear ethiology cough revealed $10 \mathrm{~cm}$ diameter aneurysm of descending thoracic aorta (TAA) and additionally $4.8 \mathrm{~cm}$ aneurysm of abdominal aorta (AAA) just below the aortic hiatus. As there was no evidence of previous trauma, Marfan syndrome or connective tissue disease patient was screened for syphilis. Diagnosis of tertiary syphilis was confirmed and specific treatment started. As complains of irritating cough intensified patients TAA was successfully treated endovascularly with thoracic stent graft (Valiant@Captivia, Medtronic Ltd.) on emergency basis. On control CT angiography 3 month after treatment there was no evidence of graft migration, endoleaks and aneurysmal sac was thrombosed. AAA has not increased in size, and open repair is planned.

Key words: syphilitic aneurysm, endovascular treatment.

\section{AIM OF THE DEMONSTRATION}

The aim of this article is to demonstrate a very rare condition - two syphilitic aortic aneurysms and successful treatment of TAA.

\section{CASE REPORT}

51 years old male with complains of chronic and increasing irritating cough underwent regular X-ray and CT exam of thorax. Performed lung CT scan was suspicious for thoracic aorta aneurysm. Patient was sent to P.Stradins Clinical University Hospital (CUH) department of vascular surgery for more extensive examination. CT angiography confirmed diagnosis of saccular TAA of $10 \mathrm{~cm}$ in diameter (picture 1) with an additional finding of $4.8 \mathrm{~cm}$ diameter aneurysm of abdominal aorta just below diaphragm (picture 2). As there was no evidence of previous trauma or connective tissue disease, patient was screened for syphilis and screening tests where positive. Final diagnosis tertiary syphilis was confirmed by Treponema pallidum hemagglutination assay (TPHA). Specific treatment was started with antibiotics - Penicillin G (for l month), but irritating cough became stronger indicating possible growth of TAA 3 weeks later. As growth of TAA could endanger patient's life due to rupture, decision to treat TAA endovascularly on emergency basis was made. The operation was performed in the vascular radiology laboratory under general anaesthesia. The right common iliac artery approach through the $5 \mathrm{~cm}$ incision parallel to the inguinal ligament was used to insert a stent graft delivery system. After applying 5000 units of heparin steerable 0.035 guidewire Back-Up Meier (Boston Scientific Scimed. Natick, MA) was inserted through a Pigtail catheter (Merit Medical OEM, South Jordan, UT) and positioned in the aortic arch. Percutaneous access to the left common femoral artery for angiographic control was gained through a 6 Fr sheath. The pig-tail catheter was inserted over a Hydrophilic 0.035 Terumo Glidewire (Terumo Interventional Systems. Somerset, NJ) and positioned in the aortic arch near the aortic valve. Over the Back-Up Meier guidewire $40 \times 40 \times 150 \mathrm{~mm}$ Valiant ${ }^{\circ}$ Captivia Thoracic Stent graft (Medtronic Ltd.) delivery system was introduced and positioned in the aortic arch via $24 \mathrm{Fr}$ sheath system (picture 3). An aortography of the arch was performed through the pig-tail catheter to ensure correct positioning and the stent graft was successfully deployed just distally from the origin of left subclavian artery (picture 4). The pigtail catheter was removed and left common iliac artery was closed using Angio Seal (St. Jude Medical. Minnetoka, $M N)$ vascular closure device. After the removal of the delivery system right common iliac artery was closed with a 6-0 Prolene interrupted suture. Abdominal aortic aneurysm was left untreated at this time.

The patient stayed for 24 hours in the intensive care unit ICU and was discharged from the hospital 4 days after the operation. Patient finished antibacterial treatment and a control CT angiography was performed after 3 months (picture 5). There were no signs of endoleak, stent graft migration, fracture or perfusion of the excluded aneurysmal sac.

\section{DISCUSSION}

Present days syphilitic aneurysms are rare pathology. Only some case reports have been published in literature over the last decade. We have not found any publication regarding double syphilitic aneurysm so far in English speaking literature $(5,8)$. Tertiary syphilis usually occurs 1-10 years after the initial infection, however in some cases it can take up to 50 years. This stage is characterized 
by the formation of gummas, which are soft, tumorlike balls of inflammation known as granulomas. The more severe manifestations include neurosyphilis and cardiovascular syphilis. In a study of untreated syphilis, $10 \%$ of patients developed cardiovascular syphilis (7), $16 \%$ had gumma formation and $7 \%$ had neurosyphilis. Cardiovascular complications include syphilitic aortitis, aortic aneurysm, aneurysm of sinus of Valsalva and aortic regurgitation. Syphilis infects the ascending aorta causing aortic dilation and aortic regurgitation. Contraction of the tunica intima leads to a tree bark appearance that is wrinkly. The aortic valve dilation and subsequent insufficiency leads to diastolic regurgitation and causes hypertrophy of the left ventricle. The clinical course of these cardiovascular effects causes mediastinal encroachment and secondary respiratory difficulties (dyspnea), difficulty swallowing (dysphagia) and persistent cough because of pressure on the recurrent laryngeal nerve triggering the cough reflex, which was observed in our case. The aneurysm developed during the disease course may also rupture, leading to massive intrathoracic hemorrhage and likely death $(2,1)$, although the most likely cause of death is the heart failure resulting from aortic regurgitation. Syphilis screening tests, such as the Rapid Plasma Reagin (RPR) and Venereal Disease Research Laboratory (VDRL) tests are not completely specific, as many other conditions can cause a positive result (4). False positives on the rapid tests can be seen in viral infections (Epstein-Barr, hepatitis, varicella, measles), lymphoma, tuberculosis, malaria, Chagas Disease, endocarditis, connective tissue disease, pregnancy, intravenous drug abuse. As a result, these two screening tests should always be followed up by a more specific treponemal test. Tests based on monoclonal antibodies and immunofluorescence, including TPHA and Fluorescent Treponemal Antibody Absorption (FTA-ABS) are more specific. Because the VDRL has a high sensitivity but low specificity, it is used as a screening test. Today, treponemal FTA-ABS TPHA tests are considered confirmatory assays with high specificity (98\%) and sensitivity (5).

In our case positive TPHA test and clinically saccular and irregular size of the anurysms helped to make proper diagnosis of syphilitic aneurysm.

Ascending aorta and aortic valve is the most common location of syphilitic aneurysms in most papers published. We could not find any report about simultaneously diagnosed syphilitic aneurysms of thoracic and abdominal aorta and treatment options in such case. Usual praxis is to treat acute infection of syphilis or other infection causing aneurismal disease and then proceed with open or endovascular treatment of aneurysm $(3,6)$. However, escalation of symptoms increased risk of rupture and in this case strategy of emergency aneurysm endovascular repair was important to save patients life.

Aneurysm of abdominal aorta at the time of repair of TAA was of relatively small size so it was left untreated. Patient also had no pain in abdomen or other complains related with. AAA was unchanged on 3 month follow
CTA scans. Nevertheless, AAA has sacullar shape, there is still risk of rupture despite small aneurysm size. Unfortunately involvement of mesenterial vessels in aneurysm (truncus coeliacus and superior mesenteric artery) excludes possibility to treat patient endovascularly with currently availably endografts. Therefore we are planning open surgical repair of AAA for this patient.

\section{Conflict of interest: None}

\section{REFERENCES}

1. Bethany Goldstein, Alfio Carrocio et al. Combined open and endovascular repair of a syphilitic aortic aneurysm // J Vasc Surg 2003;38:1422-5

2. Eduard Kieffer, Laurent Chice, Fabien Kosltas, Amine Bahnini. Aneurysms of the innominate artery: Surgical treatment of 27 patients // J Vasc Surg 2001;34:222-8

3. G. Gilling-Smith. The evidence for endovascular aneurysm repair // In: J Earnshaw, John A Murie The evidence for vascular surgery. $2^{\text {nd }}$ edition, Shrewsbury, tfm Publishing Limited, 2007; 145-153

4. Larsen SA, Steiner BM, Rudolph AH. // Laboratory diagnosis and interpretation of tests for syphilis. Clin Microbiol Rev 1995;8:1-21

5. Luis R. Leon, Joseph L. Mills. Diagnosis and management of mycotic aneurysms // vasc endovasc surg 2010 44:5

6. P. Allen Hartsell et al. Clinical manifestation and management of subclavian artery aneurysms. // perspect vasc surg endovasc ther 1999; 10: 69

7. RavulJindal, MichaelJeankins. Mycoticaneurysms// In: Sinmon D. Parvin, Jonothan J. Earnshaw Rare vascular disorders. $1^{\text {st }}$ edition, Shrewsbury, tfm Publishing Limited, 2005; 237-245

8. Torsten Bossert, R.Battellini, V.Kotowitcz, V.Falk, J.F.Gummert, F.W.Mohr. Ruptured giant syphilitic aneurysmofthedescendingaortainanoctogenarian// J Card Surg 2004; 19:356-357 


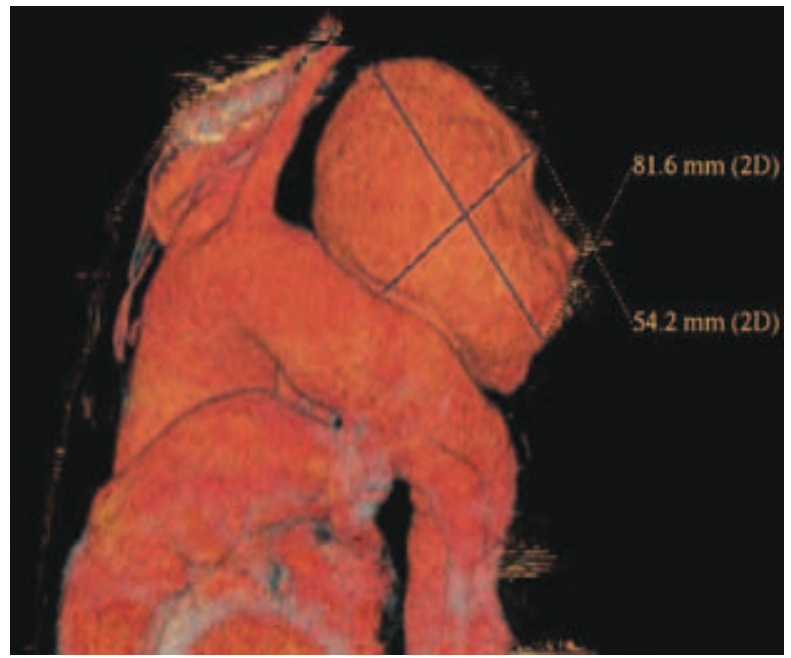

Fig. 1. Large aneurysm of descending thoracic aorta

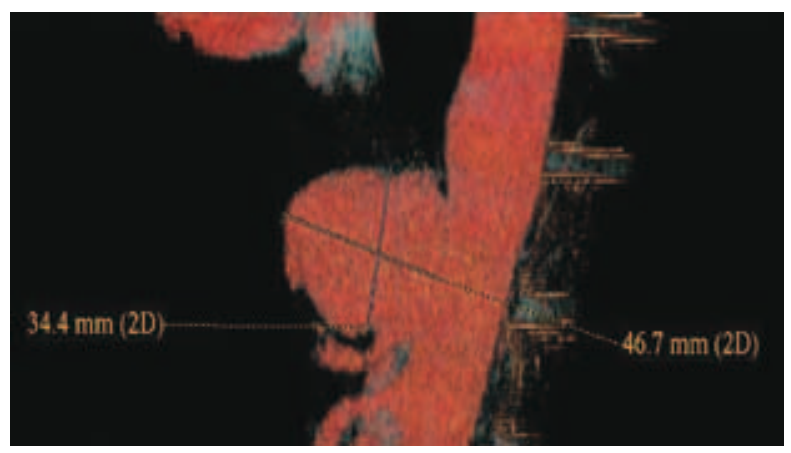

Fig. 2. Aneurysm of abdominal aorta

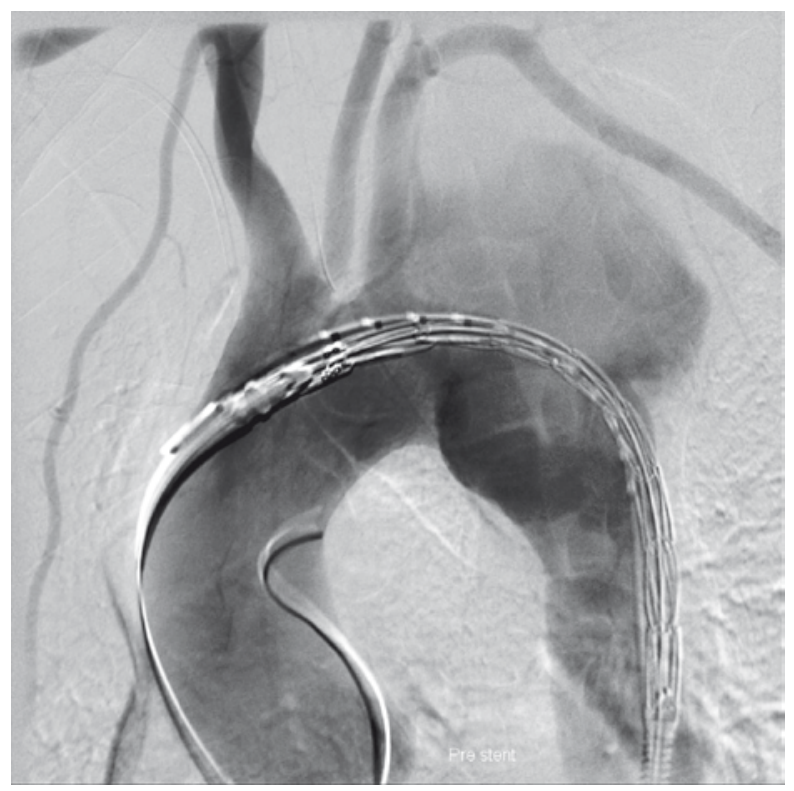

Fig. 3. Thoracic endograft placed in thoracic aorta

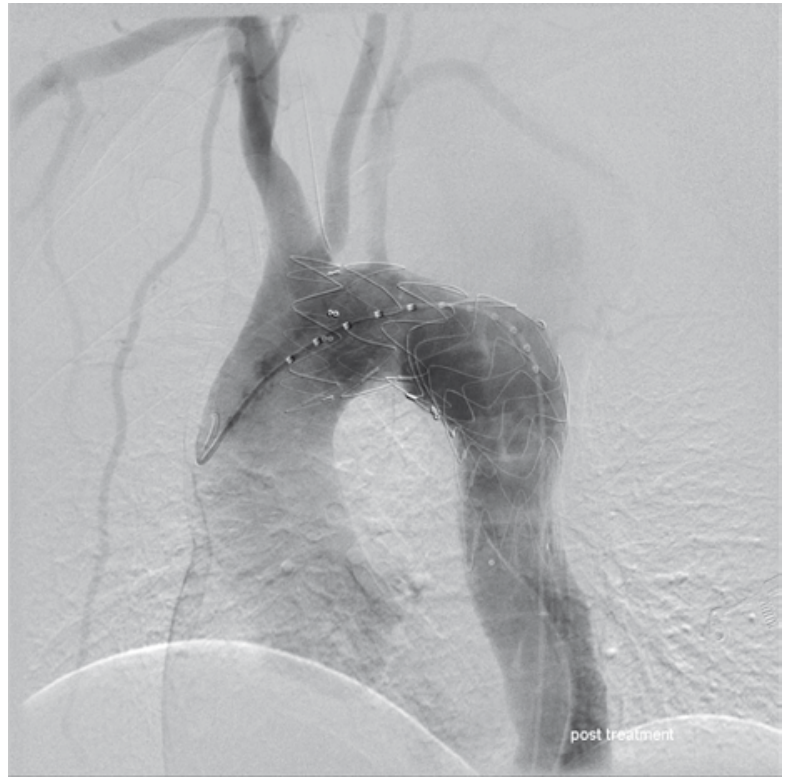

Fig. 4. Picture. Successful treatment of TAA

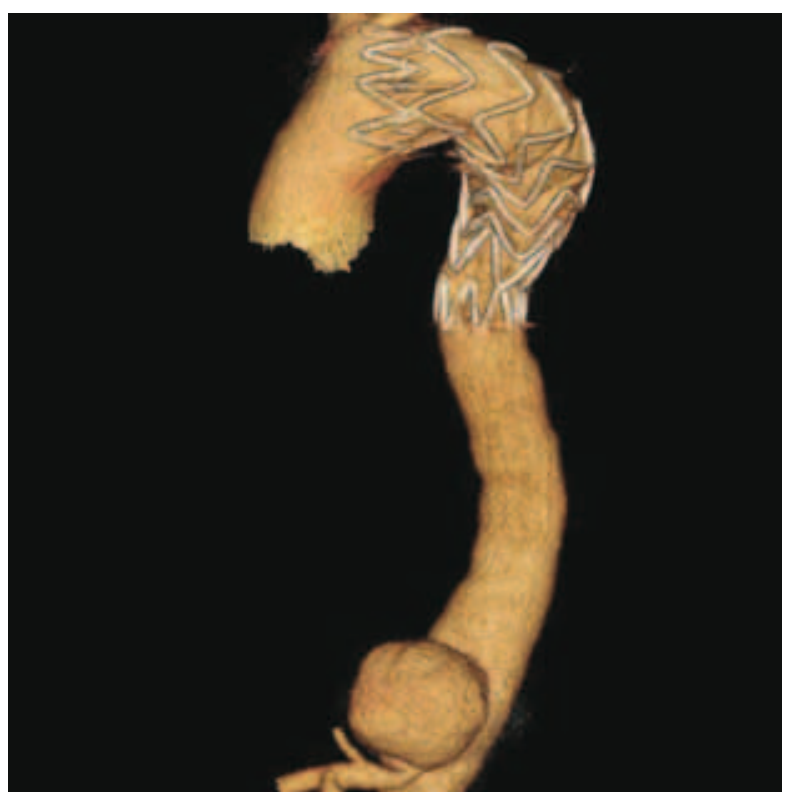

Fig. 5. Picture. 3 month follow up

\section{Address:}

Dainis Krievins

Pauls Stradins CUH, Department of Vascular surgery Pilsonu Street 13

LV-1002, Riga, Latvia

E-mail: dainis.krievins@stradini.lv 